\title{
The Effects of Interdisciplinary Bedside Rounds on Patient Centeredness, Quality of Care, and Team Collaboration: A Systematic Review
}

\author{
Tine Heip, RN, MSc, * Ann Van Hecke, RN, PhD, *广t Simon Malfait, RN, MSc, PhD, $\S$ \\ Wim Van Biesen, $M D, P h D, / / 9$ and Kristof Eeckloo, LLM, PhD*§
}

\begin{abstract}
Background: Research indicates that having multiple healthcare professions and disciplines simultaneously at the patient's bedside improves interprofessional communication and collaboration, coordination of care, and patient-centered shared decision-making. So far, no review has been conducted, which included qualitative studies, explores the feasibility of the method, and looks at differences in definitions.

Objectives: The aim of the study was to explore available evidence on the effects of interdisciplinary bedside rounds (IBRs) on patient centeredness, quality of care and team collaboration; the feasibility of IBRs; and the differences in definitions.
\end{abstract}

Data Sources: PubMed, Web of Science, and Cochrane databases were systematically searched. The reference lists of included articles and gray literature were also screened. Articles in English, Dutch, and French were included. There were no exclusion criteria for publication age or study design. Study Appraisal and Synthesis Methods: The included $(\mathrm{N}=33)$ articles were critically reviewed and assessed with the Downs and Black checklist. The selection and summarizing of the articles were performed in a 3-step procedure, in which each step was performed by 2 researchers separately with researcher triangulation afterward.

Conclusions and Implications of Key Findings: Interdisciplinary bedside round has potentially a positive influence on patient centeredness, quality of care, and team collaboration, but because of a substantial variability in definitions, design, outcomes, reporting, and a low quality of evidence, definitive results stay uncertain. Perceived barriers to use IBR are time constraints, lack of shared goals, varied responsibilities of different providers, hierarchy, and coordination challenges. Future research should primarily focus on conceptualizing IBRs, in specific the involvement of patients, before more empiric, multicentered, and longitudinal research is conducted.

Key Words: multidisciplinary, interdisciplinary, interprofessional, collaboration, patient care team, point of care systems, bed, bedside, patient participation, patient centered, family centered, patient-expert, patient-experience, decision-making, shared, round, professional practice, team collaboration, quality of care

( Patient Saf 2020;00: 00-00)

From the *Department of Public Health, Ghent University; †University Centre for Nursing and Midwifery, Ghent University; $\$$ Nursing Department, Ghent University Hospital; §Strategic Policy Unit, Ghent University Hospital; ||Department of Internal Medicine, Ghent University; and \Department of Nefrology, Ghent University Hospital, Ghent, Belgium.

Correspondence: Tine Heip, RN, MSc, Isegrimstraat 71, 9000 Ghent (e-mail: Tine.heip@ugent.be).

The authors disclose no conflict of interest.

Supplemental digital contents are available for this article. Direct URL citations appear in the printed text and are provided in the HTML and PDF versions of this article on the journal's Web site (www.journalpatientsafety.com).

Copyright (C) 2020 The Author(s). Published by Wolters Kluwer Health, Inc. This is an open-access article distributed under the terms of the Creative Commons Attribution-Non Commercial-No Derivatives License 4.0 (CCBY-NC-ND), where it is permissible to download and share the work provided it is properly cited. The work cannot be changed in any way or used commercially without permission from the journal.
D atient participation has been identified as a key aspect of patient centered care ${ }^{1}$ and patient safety. ${ }^{2}$ Healthcare systems should therefore define safety, effectiveness, and efficiency by also including measures and outcomes that are patient centered. ${ }^{3}$ This means that healthcare systems should have more attention for patients' concerns and expectations, their values, choice, their sense of dignity, and need to participate in decision-making. Doing so, the quality chasm is crossed as both healthcare workers and patients are involved and have a healthful relationship based on mutual respect and understanding, as well as shared knowledge. ${ }^{4,5}$ Achieving this is essential to even more positive health outcomes. ${ }^{3}$ Therefore, healthcare organizations are continuously searching for methods to achieve more patient centeredness. It is believed that interdisciplinary bedside rounds (IBRs), where the patient's case is discussed at the bedside, are a possible method to improve patient centeredness as well as patient safety in hospitals. ${ }^{6,7}$ Because of the communication of the care process at the bedside, the patient can participate, ask questions, or even correct false information. ${ }^{8}$

This contrasts with the current practice where the presentation of patients' cases often takes place in conference rooms or hallways. ${ }^{9,10}$ However, research indicates that having multiple disciplines and professions, at least one nurse and physician, simultaneously at the bedside improves interprofessional communication and collaboration, ${ }^{9}$ coordination of care, and patientcentered shared decision-making. ${ }^{11}$ Furthermore, patients report higher levels of satisfaction, ${ }^{12}$ and there are indications that IBR could reduce hospitalization costs ${ }^{13}$ and decrease length of stay (LOS) ${ }^{13}$ Recently, a systematic review on IBR ${ }^{14}$ was conducted, indicating that a complementary review should be conducted specifically targeting qualitative outcomes and measures concerning feasibility and systematically mapping the heterogeneity of definitions and process. Therefore, the primary goals of this review are: (1) to determine the effects of IBRs on patient centeredness, quality of care and team collaboration from both qualitative and quantitative studies; (2) to provide more insights in the heterogeneity of definitions concerning the topic; and (3) to assess the feasibility of the method. Although there is no single definition yet for IBR, ${ }^{14}$ this study considers IBR to have some minimal characteristics to relate to the introduction hereinabove. It is (1) a clinical process (2) during which caregivers from different disciplines (3) gather at the patients' bedside to (4) discuss clinical care (5) with involvement of the patient (and family), meaning that also (6) sufficient time is available to respond to questions that the patient (or family) might have.

\section{Aim}

This systematic review aims to explore (1) the effects of IBR on patient centeredness, quality of care, and team collaboration; (2) the implementation and feasibility of IBR; and (3) the heterogeneity of definitions of IBR. 


\section{METHODS}

\section{Data Sources and Searches}

A systematic literature search was conducted up to December 2018 using PubMed, Web of Science, and Cochrane databases. To identify relevant articles, a combination of relevant Medical Subject Headings, key words, and their synonyms were used (Appendix A, Supplemental Digital Content 1, http://links. lww.com/JPS/A298). Articles in English, Dutch, and French were eligible to be included. In addition, the reference lists of included articles were manually screened for additional relevant studies. In addition, an additional search of the gray literature was conducted through popular search engines like Google scholar. We also identified one ongoing clinical trial through clinicaltrials.gov. There was no age limit or exclusion because of study design to widen the scope.

\section{Study Selection}

The selection of the articles was performed in a 3-step procedure and according to the Preferred Reporting Items for Systematic Reviews and Meta-analyses guidelines. ${ }^{15}$ The initial search on academic databases identified 2261 unique titles after removing duplicates $(\mathrm{n}=2261)$. In addition, 51 articles were identified within gray literature and other sources. Of these articles $(\mathrm{n}=2312), 2223$ articles were removed based on their title and abstract. Articles in which there was only one profession focused on the use of electronic devices and aids, with a primary focus on education of healthcare workers (i.e., teaching round), and focused on children, and adolescents were excluded. Concerning the latter, communication would mainly be with family and less with the patient. Second, the remaining full texts (89) were screened in depth on 5 criteria: (1) patient centeredness; (2) quality of care; (3) team collaboration; (4) implementation and feasibility; and (5) providing a definition. Thirty-tree articles were finally withheld. A flow chart outlining the study selection process can be found in Appendix B (Supplemental Digital Content 2, http://links.lww.com/JPS/A299). Third, the Downs and Black checklist was used to assess the quality of the included articles (Appendix C, Supplemental Digital Content 3, http://links.lww. com/JPS/A300). Scoring options were yes/no/not applicable/ unable to determine $(1 / 0 /-/ 0)$ for each quality indicator. ${ }^{16}$ Studies were categorized as low (scores 0-9), medium (scores 10-18), or high (19-27). An overview of the included studies with critical appraisal and study characteristics can be found in Appendix D (Supplemental Digital Content 4, http://links.lww.com/JPS/A301). Two reviewers independently performed each of the previously mentioned steps to achieve researcher's triangulation. Discrepancies were resolved through consensus meetings between the steps.

\section{Data Collection Process}

The following study characteristics were extracted: study population, study design, and study setting. The results regarding the effect of IBR were determined for the outcomes of interest: (1) patient centeredness, (2) quality of care, and (3) team collaboration. Per study, it was determined whether these outcome measures improved, decreased, or were inconclusive because of mixed outcomes. In addition, the implementation and feasibility of IBR were mapped. Finally, we determined whether a clear definition of IBR was given, whether patients were actively involved and which healthcare workers participated. Any major flaws in the studies were identified and taken into account when reporting the results. Extraction of data from studies was independently performed by 2 reviewers. Because of variability in characteristics, research design, and outcomes, a meta-analysis was not feasible and a narrative analysis was used.

\section{RESULTS}

The included studies were conducted in the United States $(\mathrm{n}=25)$, United Kingdom $(\mathrm{n}=2)$, Scotland $(\mathrm{n}=1)$, Canada $(\mathrm{n}=1)$, Switzerland $(\mathrm{n}=1)$, and Australia $(\mathrm{n}=3)$. Researchers usually described their approach as qualitative, using individual interviews for data collection. Overall, the quality of the studies was low $(n=7)$ or medium $(n=23)$, and 3 studies were of high quality $(n=3)$. Most studies were conducted in medical or surgical wards $(n=27)$, most of them in one unit $(n=20)$, usually in a single hospital setting $(\mathrm{n}=30)$. All study characteristics can be found in Appendix D (Supplemental Digital Content 4, http:// links.lww.com/JPS/A301). A summary and overview of the findings on effect and feasibility of IBR can be found in TABLE 1 (Appendix E, Supplemental Digital Content 5, http://links.lww. com/JPS/A302)

\section{Patient Centeredness and Involvement}

As healthcare workers make the switch from performing their rounds in hallways with only short visits at the patients' bedside to bedside rounds, direct patient contacts at least doubles. ${ }^{11,12,17}$ It is hypothesized that this switch increases patient centeredness and involvement. Concerning patient centeredness, implementing IBR improves patient participation, ${ }^{17-26}$ patient empowerment, ${ }^{18,19,22,24-26}$ and had mixed effects on patient satisfaction. ${ }^{12,17-19,23,27-29}$

Being well informed, being able to express worries and fears, to discuss the treatment, and to use a shared decision-making approach, contributes to patient participation and empowerment. Patients are also more satisfied with treatment coordination, information, and discharge planning. ${ }^{20}$

Moreover, patients who are treated considerately, who understand what they are being told, and with the opportunity to ask questions, are more likely to have better clinical outcomes. ${ }^{30}$ Taking the time to talk to the patient gives caregivers the opportunity to meet with patients' unique needs. ${ }^{18}$ Patients identify such empathy and caring as an important element to address the emotional needs of patients. ${ }^{20}$ This is associated with a significant improvement of patient satisfaction with care. Moreover, proactive information provision was perceived as a relief not having to chase medical staff for information. ${ }^{18}$

\section{Quality of Care}

Performing IBR reduces adverse events, ${ }^{25,31,32,35}$ it has been shown to reduce LOS in some, ${ }^{13,29,32,33,36}$ but not all studies. $^{21,22,35,37}$ Regarding 28-day readmission rate, no effects were found. ${ }^{22,34}$ Interdisciplinary bedside round did not reduce in hospital death in elderly patients, but it may encourage patients and families to make decisions on nursing home placement earlier than they would have without IBR. ${ }^{24}$ In addition, research suggests that patients who had bedside reports spent twice as much time with their physician. ${ }^{12}$ Furthermore, studies ${ }^{23,32,38,39}$ suggest that introducing a checklist during ward rounds reduces rounding time, aids memory recall of tasks that may have otherwise been overlooked, better informs patients and their family members on current care goals, and prevents omissions. By doing this, it strengthens the effects on patient safety in an easy, costeffective way. ${ }^{38}$ Examples are the M.I.N.D.E.R.S-ward round checklist $^{38}$ and the "daily goals form.", ${ }^{32}$ All these improvements of quality of care are hypothesized to lead to decreased hospitalization costs. ${ }^{13}$ 


\section{Team Collaboration}

The highest ratings for benefits are those related to interprofessional communication ${ }^{13,26,28,31,36,38,40-44}$ and coordination. ${ }^{10,17,18,23-25,28,36,41}$ Interdisciplinary bedside rounds improve communication and therefore teambuilding between nurses and physicians and other professionals included in the rounds. ${ }^{10,13,21,24,40-44}$ To achieve this, it is however important that the shared goals are clear for the attending healthcare providers. Clarity enables staff understanding regarding to the plan of care, the ability to address patient fears and worries, staff efficiency and patient safety, and staff perceptions of care quality. ${ }^{42}$ Improving the level of understanding (i.e., situation awareness) enables correct decisions and planning because of the fact that each team member is sufficiently informed of the patient care plan and goals to effectively collaborate. ${ }^{10,18,23,24,32,37,42,44}$ Such situation awareness is a key component regarding IBR and therefore patient safety ${ }^{45}$ and should be made explicit. ${ }^{42}$ A checklist for evaluating and documenting team and collaborative behaviors during the rounds, the Bedside Rounds Checklist, is at hand to achieve this goal. ${ }^{39}$

In addition, IBR thrives best in a culture, which welcomes input from every multidisciplinary team member and patients in the decision-making process and which regards these as equal. ${ }^{33}$ Often, hierarchy negatively affects effective and safe communication. ${ }^{42}$ One common message minimizes confusion between doctors and nursing staff. This results in fewer phone calls between nurses and physicians, ${ }^{25}$ improves coordination of care and efficiency, ${ }^{25,42}$ and provides clarity to patient and family. ${ }^{25}$

Furthermore, it is important to explicitly define the role of a nurse in the process of IBR. This provides structure for participating nurses and is therefore valued. ${ }^{38}$ The collaborative nature of the rounds gives a better understanding of each team members' contribution, which results in a greater sense of mutual respect and trust. ${ }^{18}$ This enhanced team collaboration is claimed to increase job satisfaction and decrease job turnover. ${ }^{33}$

\section{Implementation and Feasibility}

Research suggests that coordination challenges ${ }^{26,28}$ and time constraints $^{10,19,26,46}$ form the biggest barriers for applying IBR in practice. Only one study concluded that introducing IBR actually resulted in significantly shorter rounds, which was enhanced by using a rounding checklist to aid in structured patient presentation. ${ }^{23}$ According to the authors, these shorter rounds could be the result of 2 factors: (1) a decrease in the time for patient presentation and (2) a reduction in interruption time. Increased time was spent during IBR, however, when there were less experienced physicians attending, when it was a weekday, and when no resident clinician was present. ${ }^{46}$

Another mentioned barrier is the inconsistency in the attendance by healthcare providers. ${ }^{41,43,46}$ For patients, it is important that a team shows up reliably and at a regular time point. ${ }^{18}$ Specifically, the timing of medication administration forms a major obstacle for IBR as nurses are often engaged in their busiest activity of the day in the middle of attending rounds. ${ }^{44}$ For the same reason, planning rounds at a time patients' needs are high (e.g., before lunch) could result in less consistent attendance of nurses. ${ }^{43}$

In addition, to make IBR possible, teams must overcome hierarchical barriers, as these hierarchies often diminish effective and safe communication. ${ }^{20,23}$ Therefore, changes made by introducing IBR are also a key step toward a culture, which welcomes input from every multidisciplinary team member and patients in the decision-making process. ${ }^{33}$
Next to healthcare worker related barriers, studies also report patient-related barriers. The process of IBR could induce stress in some patients, ${ }^{12}$ confusion could be generated by use of medical jargon, and confidentiality breaches in semiprivate rooms could occur. ${ }^{10,19}$

The feasibility of IBR is positively influenced by teams with senior residents. Factors influencing the occurrence of IBR were as follows: the experience level of the attending physician, whether IBR was performed during the week or the weekend, and the size of the team available to perform IBR. ${ }^{46}$

\section{Interdisciplinary Bedside Round Design and Definition}

Only $33 \%$ of the articles $(n=11)$ actually provided a definition of IBR, and even then, these definitions varied. Patient involvement was an essential element in $36 \%$ of the articles $(n=12)$. In $24 \%$ of the articles $(n=8)$, the rounds were conducted by a nurse and physician. In $64 \%(n=21)$, the rounds were conducted by a nurse, physician, and at least one other discipline (e.g., pharmacist, social worker, dietician, etc). In $12 \%$ of the articles $(n=4)$, the characteristics of participants were ill-defined. An overview of these findings can be found in Table 2 (Appendix F, Supplemental Digital Content 6, http://links.lww.com/JPS/A303).

\section{DISCUSSION}

This review found substantial variability in IBR definitions, design, outcomes, and reporting. Interdisciplinary bedside round has potentially a positive influence on patient centeredness, quality of care, and team collaboration and on performance measures such as LOS and patient satisfaction. However, evidence was overall of low quality and inconsistent.

Most studies did not provide a definition of IBR, and among those that did, a significant variability was found. When setting up the design of IBR, it is essential to use a clear and uniform definition for the concept to advance as a topic with a coherent, theoretically informed and practice-embedded framework. The lack of such framework could be the explanation of the mixed results. Particular attention should be given to the involvements of patients during these bedside rounds. Including the patients could have a significant positive impact on patient centeredness, patient participation, patient empowerment, and quality of care, especially from the patient's perspective. To achieve this, patients should be involved by encouraging them to participate, adding comments, and posing questions either during or before the IBR. ${ }^{20}$ Therefore, sufficient time should be available to respond to any questions that the patient (or family) might have. It is also advised to use adjusted language so that the patient understands what is being said. ${ }^{18,20}$ Making sure that the patients can identify everybody in their care team decreases the threshold to participate. ${ }^{17}$ Including and informing the patients, resulting in a better comprehension of their problem, will possibly lead to greater adherence to the suggested therapy and will induce more trust by knowing their healthcare providers. ${ }^{39}$

The composition of the interdisciplinary team in the study should also be considered. It seems logical that at minimum, a nurse and a doctor should be at the bedside in IBR. However, research suggests added value for having also other disciplines at the bedside, according to patients' needs. ${ }^{36,47}$

Perceived barriers to adequately use IBR are time constraints, lack of shared goals, varied responsibilities of different providers, hierarchy, and coordination challenges. In contrast, some studies suggest that these constraints are perhaps unfounded and based on wrong perceptions. ${ }^{23}$ Factors that can facilitate the use of IBR are incorporating structure in the rounds, for instance by using a 
checklist or script, and clear arrangements between the participating healthcare workers. ${ }^{39}$ Such interventions smoothen the process and give clarity as to which role every participant performs during the rounds. This makes participants feel more comfortable during the IBR. In addition, such a checklist can ensure that all aspects of care are included in the rounds.

Because of the potential of IBR on team communication, coordination, teambuilding, job satisfaction, and situation awareness, IBR should be further explored in multicentered, longitudinal studies as the heterogeneity observed in our review can potentially be explained by the fact that most the studies was conducted in one unit and in a single hospital.

\section{Strengths and Limitations}

We used a structured search strategy, and manual and in-depth searches for gray literature were used, making this systematic review methodologically sound. Our major finding of the lack of a consistent definition for IBR at the same time also complicates making conclusions on advantages and barriers of it. Next to the fact that differences in composition and patient involvement could have an impact on the outcomes used in this study, such unclarities could have made our inclusion process less performant. In addition, IBR was often implemented in combination with other interventions. This makes it unclear whether the reported outcomes originated because of the implementation of IBR or of the other interventions. Few publications of negative studies were found, which potentially biased our review toward the positive impact of IBR. Last, the data used in this systematic review date back to 1998 , and the review is thus quite exhaustive. Although this could be seen as a strength because of exhaustiveness, it could also be considered a limitation. Given the rapid changes to care processes in hospital settings, the results found in these older studies may be less applicable to current-day healthcare.

\section{Future Research}

Future research on IBR should start by providing a standard definition of IBR and describe the specific characteristics of the rounds. Without a common language, a wide variation in design and outcomes will remain, making it impossible to further study the method. Only after that, the IBR method can be studied and research should primarily pay specific attention to the optimal composition and size of an IBR team. In this study, IBR was defined as (1) a clinical process (2) during which caregivers from different disciplines (3) gather at the patients' bedside to (4) discuss clinical care (5) with involvement of the patient (and family), meaning that also (6) sufficient time is available to respond to questions that the patient (or family) might have. In addition, research is warranted to examine ways to actively involve patients and families. Because most research was executed in the United States, more studies in Europe should be conducted. Finally, most research was conducted in a single setting. To better evaluate the feasibility and effectiveness of IBR, prospective interventional longitudinal studies are needed. To determine time, cost, adverse events, and effect size, a pilot study must be undertaken as a first step to such a larger multicentered study.

\section{CONCLUSIONS}

Quality of evidence is hampered by a lack of one standardized definition and therefore use of IBR. To determine its effectiveness and feasibility, it is essential that a clear and preferentially uniform definition is used in the future, which is afterward used in largescale studies. Only by providing such a definition, this concept can advance as a coherent theoretically informed and practiceembedded framework. There is evidence, although low of quality, that IBR could result in an improvement of patient centeredness, quality of care, and team collaboration, at least when conducted in a structured fashion. Although patient centeredness has been identified as a key aspect of IBR, little research has been conducted regarding this topic. The highest rated barriers for implementing IBR were time constraints, coordination challenges, inconsistency in attendance of healthcare providers, hierarchical barriers, a lack of clear team goals, and role unclarity.

\section{REFERENCES}

1. Oxelmark L, Ulin K, Chaboyer W, et al. Registered nurses' experiences of patient participation in hospital care: supporting and hindering factors patient participation in care. Scand J Caring Sci. 2018;32:612-621.

2. Longtin Y, Sax H, Leape LL, et al. Patient participation: current knowledge and applicability to patient safety. Mayo Clin Proc. 2010;85:53-62.

3. Institute of Medicine. Crossing the Quality chasm: a new health system for the 21st century. Washington, DC: National Academy Press; 2001.

4. Castro EM, Van Regenmortel T, Vanhaecht K, et al. Patient empowerment, patient participation and patient-centeredness in hospital care: a concept analysis based on a literature review. Patient Educ Couns. 2016;99: 1923-1939.

5. Dewing J, McCormack B. Editorial: tell me, how do you define person-centredness? J Clin Nurs. 2017;26:2509-2510.

6. Gregory S, Tan D, Tilrico M, et al. Bedside shift reports: what does the evidence say? J Nurs Admin. 2014;44:541-545.

7. Malfait S, Eeckloo K, Lust E, et al. Feasibility, appropriateness, meaningfulness and effectiveness of patient participation at bedside shift reporting: mixed-method research protocol. J Adv Nurs. 2017;73:482-494.

8. Anderson CD, Mangino RR. Nurse shift report: who says you can't talk in front of the patient? Nurs Adm Q. 2006;30:112-122.

9. Gonzalo JD, Masters PA, Simons RJ, et al. Attending rounds and bedside case presentations: medical student and medicine resident experiences and attitudes. Teach Learn Med. 2009;21:105-110.

10. Gonzalo JD, Kuperman E, Lehman E, et al. Bedside interprofessional rounds: perceptions of benefits and barriers by internal medicine nursing staff, attending physicians, and housestaff physicians. J Hosp Med. 2014; 9:646-651

11. Gonzalo JD, Himes J, McGillen B, et al. Interprofessional collaborative care characteristics and the occurrence of bedside interprofessional rounds a cross-sectional analysis. BMC Health Serv Res. 2016;16:459.

12. Lehmann LS, Brancati FL, Chen MC, et al. The effect of bedside case presentations on patients' perceptions of their medical care. $N$ Engl J Med. 1997;336:1150-1156.

13. Curley C, McEachern JE, Speroff T. A firm trial of interdisciplinary rounds on the inpatient medical wards: an intervention designed using continuous quality improvement. Med Care. 1998;AS4-AS12.

14. Ratelle JT, Sawatsky AP, Kashiwagi DT, et al. Implementing bedside rounds to improve patient-centred outcomes: a systematic review. BMJ Qual Saf. 2019;28:317-326.

15. Liberati A, Altman DG, Tetzlaff J, et al. The PRISMA statement for reporting systematic reviews and meta-analyses of studies that evaluate health care interventions: explanation and elaboration. PLoS Med. 2009; 6:e1000100.

16. Downs SH, Black N. The feasibility of creating a checklist for the assessment of the methodological quality both of randomised and non-randomised studies of health care interventions. $J$ Epidemiol Community Health. 1998;52:377-384.

17. Luthy C, Francis Gerstel P, Pugliesi A, et al. Bedside or not bedside: evaluation of patient satisfaction in intensive medical rehabilitation wards. PLoS One. 2017;12:e170474. 
18. Uhlig PN, Brown J, Nason AK, et al. John M. Eisenberg patient safety awards. System innovation: concord hospital. Jt Comm J Qual Improv. 2002;28:666-672.

19. Chow MY, Nikolic S, Shetty A, et al. Structured Interdisciplinary Bedside Rounds in an Australian tertiary hospital emergency department: Patient satisfaction and staff perspectives. Emerg Med Australas. 2019;31:347-354.

20. Burdick K, Kara A, Ebright P, et al. Bedside interprofessional rounding: the view from the patient's side of the bed. J Patient Exp. 2017;4:22-27.

21. Dunn AS, Reyna M, Radbill B, et al. The impact of bedside interdisciplinary rounds on length of stay and complications. J Hosp Med. 2017;12:137-142.

22. Huynh E, Basic D, Gonzales R, et al. Structured interdisciplinary bedside rounds do not reduce length of hospital stay and 28-day re-admission rate among older people hospitalised with acute illness: an Australian study. Aust Health Rev. 2018;41:599-605.

23. Cao V, Tan LD, Horn F, et al. Patient-centered structured interdisciplinary bedside rounds in the medical ICU. Crit Care Med. 2018;46:85-92.

24. Basic D, Huynh E, Gonzales R, et al. Structured interdisciplinary bedside rounds, in-hospital deaths, and new nursing home placements among older inpatients. Clin Interv Aging. 2018;13:2289-2294.

25. Rimmerman CM. Establishing patient-centered physician and nurse bedside rounding. Physician Exec. 2013;39:22-25.

26. Beaird G, Dent JM, Keim-Malpass J, et al. Perceptions of teamwork in the interprofessional bedside rounding process. J Healthc Qual. 2017;39:95-106.

27. O'leary KJ, Killarney A, Hansen LO, et al. Effect of patient-centred bedside rounds on hospitalised patients' decision control, activation and satisfaction with care. BMJ Qual Saf. 2016;25:921-928.

28. Malec A, Mørk A, Hoffman R, et al. The care team visit: approaching interdisciplinary rounds with renewed focus. J Nurs Care Qual. 2018;33:135-142.

29. Begue A, Overcash J, Lewis R, et al. Retrospective study of multidisciplinary rounding on a thoracic surgical oncology unit. Clin J Oncol Nurs. 2012;16:E198-E202.

30. Department of Health, NHS. Creating a patient led NHS: delivering the NHS improvement Plan. Available at: https:/www.plymouth.ac.uk/ uploads/production/document/path/1/1921/dh_4106507.pdf. Accessed March 21, 2020.

31. Cardarelli M, Vaidya V, Conway D, et al. Dissecting multidisciplinary cardiac surgery rounds. Ann Thorac Surg. 2009;88:809-813.

32. Pronovost $\mathrm{P}$, Berenholtz $\mathrm{S}$, Dorman $\mathrm{T}$, et al. Improving communication in the ICU using daily goals. J Crit Care. 2003;18:71-75.

33. Jain M, Miller L, Belt D, et al. Decline in ICU adverse events, nosocomial infections and cost through a quality improvement initiative focusing on teamwork and culture change. BMJ Qual Saf. 2006;15:235-239.
34. Bhamidipati VS, Elliott DJ, Justice EM, et al. Structure and outcomes of interdisciplinary rounds in hospitalized medicine patients: a systematic review and suggested taxonomy. J Hosp Med. 2016;11:513-523.

35. Dutton RP, Cooper C, Jones A, et al. Daily multidisciplinary rounds shorten length of stay for trauma patients. J Trauma Acute Care Surg. 2003; 55:913-919.

36. Cornell P, Townsend-Gervis M, Vardaman JM, et al. Improving situation awareness and patient outcomes through interdisciplinary rounding and structured communication. J Nurs Adm. 2014;44:164-169.

37. Shaughnessy L, Jackson J. Introduction of a new ward round approach in a cardiothoracic critical care unit. Nurs Crit Care. 2015;20:210-218.

38. Henneman EA, Kleppel R, Hinchey KT. Development of a checklist for documenting team and collaborative behaviors during multidisciplinary bedside rounds. J Nurs Admin. 2013;43:280-285.

39. Montague M-L, Lee M, Hussain S. Staff attitudes to a daily otolaryngology ward round. J Laryngol Otol. 2004;118:963-971.

40. Urisman T, Garcia A, Harris HW. Impact of surgical intensive care unit interdisciplinary rounds on interprofessional collaboration and quality of care: Mixed qualitative-quantitative study. Intensive Crit Care Nurs. 2018; 44:18-23.

41. Gausvik C, Lautar A, Miller L, et al. Structured nursing communication on interdisciplinary acute care teams improves perceptions of safety, efficiency, understanding of care plan and teamwork as well as job satisfaction. J Multidiscip Healthc. 2015;8:33-37.

42. Prystajecky M, Lee T, Abonyi S, et al. A case study of healthcare providers' goals during interprofessional rounds. J Interprof Care. 2017; 31:463-469.

43. Young E, Paulk J, Beck J, et al. Impact of altered medication administration time on interdisciplinary bedside rounds on academic medical Ward. J Nurs Care Qual. 2017;32:218-225.

44. Mackintosh N, Berridge EJ, Freeth D. Supporting structures for team situation awareness and decision making: insights from four delivery suites. J Eval Clin Pract. 2009;15:46-54.

45. Gonzalo JD, Wolpaw DR, Lehman E, et al. Patient-centered interprofessional collaborative care: factors associated with bedside interprofessional rounds. J Gen Intern Med. 2014;29:1040-1047.

46. Kucukarslan SN, Peters M, Mlynarek M, et al. Pharmacists on rounding teams reduce preventable adverse drug events in hospital general medicine units. Arch Intern Med. 2003;163:2014-2018.

47. Hardeman W, Sutton S, Griffin S, et al. A causal modelling approach to the development of theory-based behaviour change programmes for trial evaluation. Health Educ Res. 2005;20:676-687. 